\title{
Autoconcepto de los Estudiantes del Tercer Ciclo E.E.B. - CREP. Año Escolar 2019
}

\author{
Diana Alicia Martínez \\ di.aliciamartinez@gmail.com
}

\section{RESUMEN}

El autoconcepto hace referencia a aspectos cognitivos o de conocimiento en la vida de las personas, en tal sentido este trabajo realiza un estudio descriptivo proporcionando las características de los procesos de maduración del ser humano, a partir de diferentes teorías existentes. En la adolescencia, se experimentan múltiples inestabilidades y temores propias que influyen significativamente en el desarrollo de la personalidad. Se adopta como estrategia metodológica un enfoque cuantitativo, con diseño observacional, transversal para la descripción del fenómeno, los datos son analizados mediante procedimientos de estadística descriptiva. Tiene por objetivo, evaluar la dimensión del autoconcepto de los alumnos del tercer ciclo $\left(7^{\circ} 8^{\circ}\right.$ y $\left.9^{\circ}\right)$ de la E.E.B del Centro Regional de Educación Mariscal Francisco Solano López - Pilar Paraguay. El instrumento utilizado, es el cuestionario AF- 5, apreciando las dimensiones (académico/ profesional, social, emocional, familiar y físico) variables categorizadas. Para la muestra se utilizó el $100 \%$ población, compuesta por 349 estudiantes. Se consolida a través de los resultados obtenidos que los adolescentes presentan cambios en la dimensión académica y física.

Palabras Clave: Dimensiones Autoconcepto, Adolescentes. 


\title{
Self-concept of Third Cycle Students E.E.B. - CREP. AF -5. School Year 2019
}

\begin{abstract}
Self-concept refers to cognitive or knowledge aspects in people's lives, in this sense this work carries out a descriptive study providing the characteristics of human maturation processes, based on different existing theories. In adolescence, multiple instabilities and own fears are experienced that significantly influence the development of the personality. A quantitative approach is adopted as a methodological strategy, with an observational, cross-sectional design for the description of the phenomenon, the data are analyzed using descriptive statistics procedures. Its objective is to evaluate the dimension of the selfconcept of third cycle students (7th, 8th and 9th) of the E.E.B of the Regional Center for Education Mariscal Francisco Solano López - Pilar Paraguay. The instrument used is the AF-5 questionnaire, assessing the dimensions (academic / professional, social, emotional, family and physical) categorized variables. For the sample, the $100 \%$ population was used, composed of 349 students. It is consolidated through the results obtained that adolescents present changes in the academic and physical dimension.
\end{abstract}

Keywords: Self-concept dimensions, Adolescents.

Artículo recibido: 19 enero 2020 Aceptado para publicación: 22 febrero Correspondencia: di.aliciamartinez@gmail.com Conflictos de Interés: Ninguna que declarar 


\section{INTRODUCCIÓN}

La importancia del autoconcepto ocupa un valor muy importante en la formación de la personalidad, e influye en gran medida en la autoestima. La valoración que uno mismo se da, tiene trascendencias en la autorrealización a lo largo de la vida.

Como menciona Cazalla \& Molero sobre el autoconcepto:

"se considera una necesidad humana profunda y poderosa, básica para la vida sana, con un buen funcionamiento y para la autorrealización. Está relacionado con nuestro bienestar en general. Muchos de los problemas psicológicos actuales como la depresión o los malos tratos conyugales se relacionan con un autoconcepto bajo o defectuoso" (Cazalla Luna \& Molero, 2013, pág. 44)

La pre adolescencia es el "paso" entre la niñez y la adolescencia y se caracteriza porque crea un desequilibrio emocional con conductas frecuentemente contradictorias, lo que la hace especialmente desconcertante tanto para los menores como para los padres. (Marulanda, 1999, pág. 221) A esto también se suman las manifestaciones de cambios físicos propios de la maduración. Por lo general se vive y se percibe a los adolescentes como un problema, pocas veces se les valora como individuos que transitan hacia la edad adulta y que viven un momento de grandes cambios e incertidumbres. La adolescencia es un ciclo donde minan los cambios, con sentimientos de confusión, angustia y desasosiego, esta etapa es caracterizada por las crisis más fuertes que atraviesa el ser humano. (Reigal Garrido, Videra García, Parra Flores, \& Juárez Ruiz de Mier, 2012)

Se pretende obtener acorde a los objetivos trazados en esta investigación, globalmente la percepción sobre las dimensiones del Autoconcepto en los adolescentes escolarizados en el tercer ciclo de la institución educativa del CREP.

\section{Antecedentes}

El autoconcepto en la adolescencia, como refieren en su investigación, (Antolín Suárez, Oliva Delgado, Pertegal Vega, \& López Jiménez, 2011) La visión negativa de la adolescencia predominante en la psicología a lo largo del siglo XX llevó a un mayor interés por el estudio de las conductas problemáticas, que tuvo como efecto secundario una menor inversión en recursos para el estudio y la promoción de comportamientos positivos y saludables, así como para la creación y validación de instrumentos para evaluarlos (Oliva, y otros, 2010) 
Los cuestionarios de Autoconcepto Forma-A (AFA), de Musitu et al. (1994) y el Autoconcepto Forma-5 (AF-5), de García y Musitu (2001), construidos originariamente en lengua castellana y de gran uso en nuestro entorno, incluyen el autoconcepto social como un dominio independiente dentro del autoconcepto general.

Algunos cuestionarios, como el Social Self-Invetory -SSEI- (Lawson, Mashall y McGrath, 1979) y el Social Self-Concept Scale-SSCS- (Zorich, y Reynolds, 1988), se destinan a medir únicamente el autoconcepto social, pero no reúnen buenas propiedades psicométricas.

En estos trabajos citados se aprecia un predominio de la tendencia a diferenciar el autoconcepto social por contextos (Byrne y Shavelson, 1996; Shavelson et al. 1976; Song y Hattie, 1984) al asumirse que la autopercepción como ser social varía en función de las interacciones en los distintos contextos en los que el ser humano actúa (por ejemplo, con los pares, con personas del mismo o de otro sexo, con los padres, los hermanos, la familia...). En este sentido, el autoconcepto representaría la percepción que cada cual tiene de sus habilidades sociales con respecto a las interacciones sociales con los otros y se calcula a partir de la autovaloración del comportamiento en los diferentes contextos sociales (Markus y Wurf, 1987; Vallacher y Wegner, 1987)

Hacia fines del siglo XIX, y ya en el campo de la Psicología, James (1890; citado en Casullo, 1990) consideró no sólo la imagen que uno tiene de sí mismo sino la que otros tienen de uno, sentando las bases para el estudio del autoconcepto desde una concepción psicosocial. James se refirió a un "sí mismo material" (preocupado por objetos y lugares), un "sí mismo social" (preocupado por relaciones e interacciones) y un "sí mismo espiritual" (preocupado por especulaciones concernientes a la realidad inmediata). Baldwin (1897) y Cooley (1902) (citados en Díaz-Loving, 2005) se ocuparon del aspecto evolutivo del "yo" y consideraron que el "sí mismo" es resultado de la interacción del individuo con su medio social. Si bien Freud (1919; citado en Casullo, 1990) no se abocó específicamente a esta temática, el autoconcepto queda implícito en el concepto de "yo" como una de las tres estructuras básicas que componen la personalidad (yo, ello y superyó).

De acuerdo a lo denominado Desarrollo Positivo Adolescente (DPA), prevención no es sinónimo de promoción, y una adolescencia saludable y adecuada requiere de algo más que la evitación de algunos comportamientos como la violencia, el consumo de drogas o 
las prácticas sexuales de riesgo; precisan de la consecución por parte del chico o la chica de una serie de competencias, valores y habilidades adecuadas que le permitan una exitosa transición hacia la etapa adulta. (Antolín Suárez, Oliva Delgado, Pertegal Vega, \& López Jiménez, 2011)

Hoy día la educación es considerada un espacio donde el papel prioritario es la formación de moral y socioemocional, si bien es cierto, los estudiantes ya adquieren en sus familias conductas de convivencia y hábitos, la escuela es propicia acciones que influyen en el individuo. Las relaciones y competencias permiten que los estudiantes puedan afrontar con éxito su vida personal y profesional.

Así, durante las últimas décadas, la investigación y aplicación de programas de educación para el desarrollo emocional y social, que incluyen la formación en valores, han ido en aumento en diversos países, y disponer de adecuados instrumentos de evaluación se constituye como un objetivo prioritario

\section{Planteamiento del Problema}

El presente trabajo tiene como finalidad analizar los niveles de autoconcepto y el grado de conocimiento de los estudiantes adolescentes.

Tanto el autoconcepto como las expectativas influyen en el ámbito escolar. La evidencia indica que ambas se relacionan con las atribuciones que hacen las personas

A lo largo de la vida, las personas atraviesan momentos especiales e influyentes en su desenvolvimiento social, uno de los periodos de la etapa evolutiva más compleja es la adolescencia, donde los ajustes y los nuevos cambios generan contraposición.

Se pretende comprobar el autoconcepto en las diferentes dimensiones de los alumnos del tercer ciclo del Centro Regional de Educación. "Mcal. Francisco Solano López", de la ciudad de Pilar, año 2019.

De esta forma, el nivel en los reactivos de las dimensiones que mide el instrumento administrado a la mencionada muestra, manifiesta la importancia que guarda el autoconcepto en esta etapa del desarrollo evolutivo, con varios cambios, sean éstos culturales y sociales, considerando a la misma como un factor multifacética, que ayuda a categorizar e incluir áreas tales como la escuela, la aceptación social, el atractivo físico y habilidades sociales y físicas, que junto con el factor afectivo emocional van construyendo y formando el autoconcepto. 


\section{ESTRATEGIAS METODOLÓGICAS O MATERIALES Y MÉTODOS}

El trabajo de investigación corresponde a la metodología de tipo descriptivo, no experimental, comprende la descripción, registro, análisis e interpretación de la naturaleza actual, y la composición o proceso de los fenómenos de las dimensiones de la AF-5 (García \& Musitu, AF-5 Autoconcepto Forma 5. , 1999).

El enfoque se hace sobre conclusiones dominantes o sobre grupo de personas, grupo o cosas, se conduce o funciona en presente (Tamayo y Tamayo, 2003) De enfoque cuantitativo, el cual parte de una idea que va acotándose y, una vez delimitada, se derivan objetivos y preguntas de investigación, se revisa la literatura y se construye un marco o una perspectiva teórica. De corte transversal que analiza datos de variables recopiladas en un período de tiempo sobre una población, muestra o (Hernández Sampieri, 2014). subconjunto predefinido la población está compuesta por un total de 349 estudiantes del Tercer Ciclo del CREP “Mcal. Francisco Solano López”, de la ciudad de Pilar- Paraguay, año 2019, distribuida en 192 estudiantes del sexo femenino y 157 estudiantes del sexo masculino, de entre 12 a 15 años de edad, para la muestra se utilizó el total de la población.

\section{Instrumentos}

Se administró a los estudiantes del tercer ciclo $\left(7^{\circ} 8^{\circ}\right.$ y $\left.9^{\circ}\right)$ la escala de 30 ítems discriminadas en cinco dimensiones AF-5 (García \& Musitu, AF-5 Autoconcepto Forma 5. , 1999) (académico, social, emocional, familiar y físico), se fundamentan en el modelo teórico de (Shavelson , Hubner, \& Stanton, 1976) quienes, entre otras características como la organización jerárquica a partir de una dimensión general, que el autoconcepto presenta diversos aspectos relacionados no ortogonales pero distinguibles, que pueden encontrarse diferencialmente relacionados con distintas áreas del comportamiento humano (Musitu, García, \& Gutiérrez, 1994), tres dimensiones miden con 6 ítems, una dimensión presenta siete ítems y otra dimensión cinco ítems, (García \& Musitu, AF-5 Autoconcepto Forma 5. , 1999, pág. 11), los autores asignaron a priori a cada una de estas cinco dimensiones; lo cual permite, en una escala de 1 a 99, medir con un único instrumento las principales dimensiones del autoconcepto. (Bustos, Oliver, \& Galiana, 2015)

Las cinco subescalas del instrumento utilizado están conformada de la siguiente manera. Autoconcepto Académico, $(A F-1)$ es la concepción que el individuo se forma respecto de sí mismo en su rol específico como estudiante. Este resulta y toma forma a partir del 
conjunto de experiencias, vivencias y retroalimentación recibida del medio, específicamente de los otros quesean significativos para sí, experimentadas a lo largo de los años escolares y universitarios. Este autoconcepto se logra evidenciar en dos ejes: el primero se relaciona con el sentimiento que el estudiante tiene de su rol a partir de los juicios de pares y profesores (buen estudiante; en el segundo eje se ubica las cualidades específicas valoradas en ese contexto (inteligente, dedicado, buen orador, ETC). (García \& Musitu, AF-5 Autoconcepto Forma 5. , 1999).

Autoconcepto Social, $(A F-2)$ es la percepción que tiene el individuo acerca de su desempeño en las relaciones sociales definiéndola en dos dimensiones. La primera: la red social del individuo y su facilidad o dificultad para mantenerla o ampliarla. La segunda: las cualidades personales dentro de las relaciones interpersonales como, por ejemplo: la simpatía, amabilidad, entre otras.

Autoconcepto Emocional ( $A F-3$ ) hace referencia a la percepción del estado emocional del individuo y de su respuesta ante situaciones específicas. Los autores García, Herrero y Musitu (1995), indican que este factor cuenta con dos aspectos de significado, la primera refiere a la percepción general del estado emocional del individuo y la segunda a la situación particular en la que el individuo se le pide hacer alguna actividad. Siendo un autoconcepto emocional alto, el individuo quien tenga el control de las situaciones y emociones, por el contrario, quien tiene un autoconcepto bajo es quien no logra tener una estabilidad o tranquilidad emocional ante situaciones diversas. (García \& Musitu, Manual $4^{\circ}$ edición, revisada y ampliada., 2014, pág. 18)

Autoconcepto Familiar (AF - 4) es la percepción que tiene el individuo acerca de su implicación, participación e integración en el medio familiar, también se articula bajo dos ejes. El primero está involucrado con los padres respecto a las relaciones familiares como la confianza y el afecto, el segundo refiere a la familia y el hogar respecto al apoyo, inclusión, reconocimiento y aceptación que el individuo reciba, siendo este un factor altamente importante del autoconcepto. Abril (1996); Pinazo (1993); Gil (1997) y Linares (1998) citados por (García \& Musitu, Manual 4 edición, revisada y ampliada., 2014)

Autoconcepto Físico (AF - 5) refiere la percepción de aspecto físico y de su condición, como señalan (Rodriguez, Goñi, \& Ruíz de Azúa, 2006) que la vida saludable y el bienestar psicológico tienen relación con el autoconcepto físico. 
Hay que considerar que el autoconcepto es una estructura de contenidos múltiples acerca del sí mismo, internamente consistente y jerárquicamente organizada. Como expresa (Barrera Hurtado, 2012) surge de la comparación social momentánea variando notablemente en función de dicho contexto social donde se desenvuelve el individuo.

El autoconcepto es una de las variables imprescindibles en el desarrollo personal, juega un rol importante el autoconcepto en la adolescencia va asumiendo una combinación de caracteres, capacidades, conductas, actitudes y valores que en su momento lo condiciona y lo hace diferente al resto de los demás. En la definición del "quien soy yo" se configura en aspectos cognitivos y sociales, va cambiando su pensamiento y creencias a lo largo del tiempo así formándose complejamente

\section{RESULTADOS Y DISCUSIÓN}

Con base a los resultados obtenidos, se realizó una escala de valoración clasificada entre el parámetro; nivel inferior, normal y superior al término. Con la intención de distribuir las frecuencias de las respuestas de los estudiantes del tercer ciclo en las diferentes dimensiones del autoconcepto de los estudiantes del tercer ciclo CREP, año 2019.

Tabla 1. Escala de Niveles.

\begin{tabular}{|c|c|}
\hline $01-39$ & Nivel inferior \\
\hline $40-69$ & Nivel normal \\
\hline $70-99$ & Nivel superior. \\
\hline
\end{tabular}

Elaboración propia

Tabla 2. Ítems de las Dimensiones del Test de Autoconcepto de AF- 5.

\begin{tabular}{|c|c|}
\hline \multicolumn{2}{|r|}{ Dimensiones del Autoconcepto Forma 5} \\
\hline $\begin{array}{c}\text { AF - 1 } \\
\text { Dimensión } \\
\text { Académico }\end{array}$ & $\begin{array}{l}\text { Ítem 1. Hago bien los trabajos escolares } \\
\text { Ítem 6. Mis profesores me consideran un buen trabajador } \\
\text { Ítem 11. Trabajo mucho en clase } \\
\text { Ítem 16. Mis profesores me estiman } \\
\text { ítem } 21 \text { Soy un buen estudiante } \\
\text { Ítem 26. Mis profesores me consideran inteligente y trabajador. }\end{array}$ \\
\hline $\begin{array}{c}\text { AF - } 2 \\
\text { Dimensión } \\
\text { Social }\end{array}$ & $\begin{array}{l}\text { Ítem 2. Consigo fácilmente amigos } \\
\text { Ítem 7. Soy una persona amigable } \\
\text { Ítem } 8 \text {. Muchas cosas me ponen nervioso } \\
\text { Ítem 12. Es difícil para mí hacer amigos } \\
\text { Ítem 17. Soy una persona alegre } \\
\text { Ítem 22. Me cuesta hablar con desconocidos } \\
\text { Ítem 27. Tengo muchos amigos }\end{array}$ \\
\hline
\end{tabular}




\begin{tabular}{|c|c|}
\hline $\begin{array}{c}\text { AF - } 3 \\
\text { Dimensión } \\
\text { Emocional }\end{array}$ & $\begin{array}{l}\text { Ítem 3. Tengo miedo de algunas cosas } \\
\text { Ítem } 13 . \text { Me asusto con facilidad } \\
\text { Ítem } 18 \text { Cuando los mayores me dicen algo me pongo muy } \\
\text { nervioso } \\
\text { Ítem } 23 \mathrm{Me} \text { pongo nervioso cuando me pregunta el profesor } \\
\text { Ítem } 28 \mathrm{Me} \text { siento nervioso }\end{array}$ \\
\hline $\begin{array}{c}\text { AF - 4 } \\
\text { Dimensión } \\
\text { Familiar }\end{array}$ & $\begin{array}{l}\text { Ítem 4. Soy muy criticado en casa } \\
\text { Ítem 9. Me siento feliz en casa } \\
\text { Ítem 14. Mi familia está decepcionada de mí } \\
\text { Ítem 19. Mi familia me ayudaría en cualquier tipo de problemas } \\
\text { Ítem 24. Mis padres me dan confianza } \\
\text { Ítem 29. Me siento querido por mis padres }\end{array}$ \\
\hline $\begin{array}{l}\text { AF - } 5 \\
\text { Dimensión } \\
\text { Físico }\end{array}$ & $\begin{array}{l}\text { Ítem } 5 . \text { Me cuido físicamente } \\
\text { Ítem } 10 . \text { Me buscan para realizar actividades deportivas } \\
\text { Ítem } 15 . \text { Me considero elegante } \\
\text { Ítem } 20 . \text { Me gusta como soy físicamente } \\
\text { Ítem } 25 . \text { Soy bueno haciendo deporte. }\end{array}$ \\
\hline
\end{tabular}

Elaboración propia

\section{Análisis de los resultados}

Tabla 3. Variables de la muestra utilizada (7 $7^{a}$ rado) E.E.B, CREP

\begin{tabular}{|l|c|c|c|c|c|c|c|}
\hline Séptimo $\mathbf{7}^{\circ} \mathbf{1 1 8}$ alumnos & \multicolumn{4}{c|}{ Femenino (2) } \\
\hline Sexo & \multicolumn{3}{|c|}{ Masculino (1) } & \multicolumn{3}{c|}{} \\
\hline \multirow{3}{*}{ Edad } & 58 estudiantes & $49 \%$ & 60 estudiantes & \multicolumn{2}{|c|}{$51 \%$} \\
& $\mathbf{1 2}$ & $\mathbf{1 3}$ & $\mathbf{1 4}$ & $\mathbf{1 1}$ & $\mathbf{1 2}$ & $\mathbf{1 3}$ & $\mathbf{1 4}$ \\
\cline { 2 - 9 } & 32 & 23 & 3 & 1 & 43 & 14 & 2 \\
\cline { 2 - 9 } & $55 \%$ & $40 \%$ & $5 \%$ & $1 \%$ & $72 \%$ & $23 \%$ & $3 \%$ \\
\hline
\end{tabular}

Elaboración propia

Tabla 4. Distribución de resultados por escala del $7^{\circ}$ E.E.B, CREP

\begin{tabular}{|c|c|c|c|}
\hline \multicolumn{4}{|c|}{$\begin{array}{c}\text { AF } 17^{\circ} \text { GRADO } \\
\text { ítems; }(1,6,11,16,21,26)\end{array}$} \\
\hline SEXO & Inferior & Normal & Superior \\
\hline $1(\mathrm{M})$ & 10 & 27 & 21 \\
\hline $2(F)$ & 5 & 18 & 37 \\
\hline \multicolumn{4}{|c|}{$\begin{array}{c}\text { AF } 27^{\circ} \text { GRADO } \\
\text { ítems; }(2,7,8,12,17,22,27)\end{array}$} \\
\hline SEXO & Inferior & Normal & Superior \\
\hline $1(\mathrm{M})$ & 4 & 28 & 26 \\
\hline $2(F)$ & 5 & 28 & 27 \\
\hline
\end{tabular}

Ciencia Latina Revista Científica Multidisciplinar, Ciudad de México, México.

ISSN 2707-2207 / ISSN 2707-2215 (en línea), enero-febrero, 2021, Volumen 5, Número 1. https://doi.org/10.37811/cl rcm.v5i1.277 p. 806 


\begin{tabular}{|c|c|c|c|}
\hline \multicolumn{4}{|c|}{$\begin{array}{c}\text { AF } 37^{\circ} \text { GRADO } \\
\text { ítems; }(3,13,18,23,28)\end{array}$} \\
\hline SEXO & Inferior & Normal & Superior \\
\hline $1(\mathrm{M})$ & 21 & 33 & 4 \\
\hline $2(F)$ & 23 & 24 & 13 \\
\hline \multicolumn{4}{|c|}{$\begin{array}{c}\text { AF } 47^{\circ} \text { GRADO } \\
\text { ítems; }(4,9,14,19,24,29)\end{array}$} \\
\hline SEXO & Inferior & Normal & Superior \\
\hline $1(\mathrm{M})$ & 4 & 41 & 13 \\
\hline $2(F)$ & 5 & 35 & 20 \\
\hline \multicolumn{4}{|c|}{$\begin{array}{c}\text { AF } 57^{\circ} \text { GRADO } \\
\text { ítems; }(5,10,15,20,25,30)\end{array}$} \\
\hline SEXO & Inferior & Normal & Superior \\
\hline $1(\mathrm{M})$ & 8 & 21 & 29 \\
\hline $2(F)$ & 14 & 24 & 22 \\
\hline
\end{tabular}

Elaboración propia

Tabla 5. Variables de la muestra utilizada $\left(8^{\circ}\right.$ grado $)$ E.E.B, CREP

\begin{tabular}{|l|c|c|c|c|c|c|c|c|}
\hline Octavo $8^{\circ} 114$ & alumnos & \multicolumn{5}{c|}{ Femenino (2) } \\
\hline Sexo & \multicolumn{3}{|c|}{ Masculino (1) } & 62 & \multicolumn{3}{|c|}{$54 \%$} \\
\hline & 52 & \multicolumn{3}{|c|}{$46 \%$} & 12 & 13 & 14 & 15 \\
\hline Edad & 13 & 14 & 15 & 16 & 12 & 37 & 22 & 2 \\
\hline & 34 & 16 & 1 & 1 & 1 & $60 \%$ & $35 \%$ & $3 \%$ \\
\hline
\end{tabular}

\begin{tabular}{|c|c|c|c|}
\hline \multicolumn{4}{|c|}{$\begin{array}{c}\text { AF1 } 8^{\circ} \text { GRADO } \\
\text { ítems; }(1,6,11,16,21,26)\end{array}$} \\
\hline SEXO & Inferior & Normal & Superior \\
\hline $1(\mathrm{M})$ & 5 & 30 & 17 \\
\hline $2(\mathrm{~F})$ & 8 & 22 & 32 \\
\hline \multicolumn{4}{|c|}{$\begin{array}{c}\text { AF2 8 } \text { 8 }^{\circ} \text { GRADO } \\
\text { ítems; }(2,7,8,12,17,22,27)\end{array}$} \\
\hline SEXO & Inferior & Normal & Superior \\
\hline $1(\mathrm{M})$ & 6 & 23 & 23 \\
\hline $2(\mathrm{~F})$ & 5 & 35 & 22 \\
\hline \multicolumn{4}{|c|}{$\begin{array}{c}\text { AF3 8 }{ }^{\circ} \text { GRADO } \\
\text { ítems; }(3,13,18,23,28)\end{array}$} \\
\hline SEXO & Inferior & Normal & Superior \\
\hline $1(\mathrm{M})$ & 20 & 29 & 3 \\
\hline $2(\mathrm{~F})$ & 14 & 35 & 13 \\
\hline \multicolumn{4}{|c|}{ AF4 $8^{\circ}$ GRADO } \\
\hline
\end{tabular}

Ciencia Latina Revista Científica Multidisciplinar, Ciudad de México, México. 


\begin{tabular}{|c|c|c|c|}
\hline \multicolumn{4}{|c|}{ ítems; (4,9,14,19,24,29) } \\
\hline SEXO & Inferior & Normal & Superior \\
\hline $1(\mathrm{M})$ & 2 & 40 & 10 \\
\hline $2(\mathrm{~F})$ & 3 & 46 & 13 \\
\hline \multicolumn{4}{|c|}{ AF5 8 $\mathbf{~ G R A D O ~}^{\text {ítems; } \mathbf{( 5 , 1 0 , 1 5 , 2 0 , 2 5 , 3 0 )}}$} \\
\hline SEXO & Inferior & Normal & Superior \\
\hline $1(\mathrm{M})$ & 8 & 18 & 26 \\
\hline $2(\mathrm{~F})$ & 13 & 31 & 18 \\
\hline
\end{tabular}

\section{Elaboración propia}

Tabla 6. Distribución de resultados por escala del $8^{\circ}$ E.E.B, CREP

Elaboración propia

Tabla 7. Variables de la muestra utilizada $\left(9^{\circ}\right.$ grado $)$ E.E.B, CREP

\begin{tabular}{|c|c|c|c|c|c|c|c|c|}
\hline \multicolumn{9}{|c|}{ Noveno $^{\circ} 117$ alumnos } \\
\hline \multirow[t]{2}{*}{ Sexo } & \multicolumn{4}{|c|}{ Masculino (1) } & \multicolumn{4}{|c|}{ Femenino (2) } \\
\hline & \multicolumn{2}{|l|}{47} & \multicolumn{2}{|c|}{$40 \%$} & \multicolumn{2}{|l|}{70} & \multicolumn{2}{|c|}{$60 \%$} \\
\hline \multirow[t]{3}{*}{ Edad } & 13 & 14 & 15 & 16 & 14 & 15 & 16 & 17 \\
\hline & 1 & 20 & 19 & 7 & 50 & 17 & 2 & 1 \\
\hline & $2 \%$ & $43 \%$ & $40 \%$ & $15 \%$ & $71 \%$ & $24 \%$ & $3 \%$ & $2 \%$ \\
\hline
\end{tabular}

\begin{tabular}{|c|c|c|c|}
\hline \multicolumn{4}{|c|}{$\begin{array}{c}\text { AF1 } 9^{\circ} \text { GRADO } \\
\text { ítems; }(1,6,11,16,21,26)\end{array}$} \\
\hline SEXO & Inferior & Normal & Superior \\
\hline $1(\mathrm{M})$ & 5 & 27 & 14 \\
\hline $2(\mathrm{~F})$ & 7 & 30 & 33 \\
\hline \multicolumn{4}{|c|}{$\begin{array}{c}\text { AF2 }{ }^{\circ} \text { GRADO } \\
\text { ítems; }(2,7,8,12,17,22,27) \\
\end{array}$} \\
\hline SEXO & Inferior & Normal & Superior \\
\hline $1(\mathrm{M})$ & 0 & 25 & 22 \\
\hline $2(\mathrm{~F})$ & 1 & 42 & 27 \\
\hline \multicolumn{4}{|c|}{$\begin{array}{c}\text { AF3 9 }{ }^{\circ} \text { GRADO } \\
\text { ítems; }(3,13,18,23,28)\end{array}$} \\
\hline SEXO & Inferior & Normal & Superior \\
\hline $1(\mathrm{M})$ & 15 & 24 & 8 \\
\hline $2(\mathrm{~F})$ & 15 & 37 & 18 \\
\hline \multicolumn{4}{|c|}{$\begin{array}{c}\text { AF4 9 }{ }^{\circ} \text { GRADO } \\
\text { ítems; }(4,9,14,19,24,29)\end{array}$} \\
\hline SEXO & Inferior & Normal & Superior \\
\hline
\end{tabular}

Ciencia Latina Revista Científica Multidisciplinar, Ciudad de México, México. 


\begin{tabular}{|c|c|c|c|}
\hline $1(\mathrm{M})$ & 3 & 26 & 18 \\
\hline $2(\mathrm{~F})$ & 5 & 54 & 11 \\
\hline \multicolumn{4}{|c|}{$\mathbf{A F 5 ~ 9}^{\circ}$ GRADO } \\
\hline \multicolumn{4}{|c|}{ ítems; $\mathbf{( 5 , 1 0 , 1 5 , 2 0 , 2 5 , 3 0 )}$} \\
\hline SEXO & Inferior & Normal & Superior \\
\hline $1(\mathrm{M})$ & 8 & 16 & 23 \\
\hline $2(\mathrm{~F})$ & 16 & 34 & 20 \\
\hline
\end{tabular}

Elaboración propia

Tabla 8. Distribución de resultados por escala del $9^{\circ}$ E.E.B, CREP

\section{Elaboración propia}

Se precisa en los reactivos más preponderantes y predominantes, de las variables del AF5, descritas en cuanto sigue. En la DIMENSIÓN ACADÉMICA, de la muestra en el $7^{\circ}$ grado, del sexo femenino predomina los resultados en la clasificación Superior, en cambio, los estudiantes del sexo masculino señalan con mayor frecuencia el término normal en cuanto a los ítems académico $(1.6,11,16,21,26)$. En el $8^{\circ}$ grado, dentro de los indicadores los estudiantes del sexo masculino presentan mayores resultados a nivel normal, en un 58\% en tanto, las estudiantes del sexo femenino sus respuestas dan un porcentaje de $52 \%$ significativo en el nivel superior, distribuido los demás resultados en $35 \%$ normal, y $13 \%$ inferior. En el $9^{\circ}$ grado la distribución de los resultados de los ítems (1. 6, 11, 16, 21, 26), presentan una diferencia por sexo, los estudiantes del sexo masculino en un $35 \%$ han especificado dentro del nivel normal, otros $30 \%$ dentro del nivel superior y $13 \%$ inferior, en este mismo apartado, las estudiantes mujeres clasifican en un alto nivel de categoría superior, $47 \%$, otras en un $43 \%$ normal y las restantes en un $10 \%$ dentro del nivel inferior.

En la DIMENSIÓN SOCIAL, los estudiantes del $7^{\circ}$ grado, ambos sexos; masculino y femenino indican resultados similares en respuestas a los ambos predominan $28 \%$ clasificados en el término normal, el $26 \%$ del sexo masculino señala un nivel superior y $27 \%$ en el sexo femenino puntúa en el nivel superior, en esta categoría es ínfima el porcentaje interior, $4 \%$ en el sexo masculino y $5 \%$ en el sexo femenino. Los estudiantes del $8^{\circ}$ grado es su mayoría equilibran los resultados en un porcentaje de $44 \%$ nivel normal, y $44 \%$ nivel superior, y el resto de estudiantes clasifican resultados a nivel inferior en un $12 \%$. Las estudiantes del sexo femenino en un $56 \%$ sus respuestas equivalen a término medio normal, el $35 \%$ superior y $8 \%$ inferior. En tanto los alumnos del $9^{\circ}$ grado, 
dentro de la dimensión social los varones clasifican $47 \%$ dentro del nivel superior, y las mujeres en un 39\%. En el nivel normal las estudiantes del sexo femenino encajan en un $60 \%$, y los varones en un 53\%, en el nivel inferior 0\%varoes y $1 \%$ mujeres, en esta área los estudiantes adolescentes no tienen dificultades, es decir la sociabilidad y relación con sus pares forman parte del desarrollo de su personalidad y conformación de relaciones interpersonales.

En la DIMENSIÓN EMOCIONAL, Los ítems del AF- $3(3,13,18,23,28)$, en la que se refleja que los estudiantes han intentado evadir y responder solapadamente los ítems. Considerando las respuestas en conjunto, ambos sexos predominan dentro de los términos Normales. En tanto, los estudiantes del $8^{\circ}$ grado coinciden en el término normal englobando $56 \%$ sexo masculino y $56 \%$ sexo femenino. Las mujeres hacen referencia en un nivel superior $21 \%$ y los varones $6 \%$. En este aspecto los estudiantes del sexo masculino presentan un alto puntaje en el nivel inferior. Y las mujeres $23 \%$. De la muestra tomada de los estudiantes del $9^{\circ}$ grado se pudo visualizar una distribución de frecuencia, tanto varones y mujeres responden dentro del término Normal, el 26\% del sexo femenino clasifica en el nivel superior, y 17\% los varones en el nivel superior. se percibe en el nivel inferior $32 \%$ de las respuestas de los varones encasillados en el rango inferior, y $21 \%$ las mujeres en el mismo rango.

La DIMENSIÓN FAMILIAR, Los ítems evaluados AF 4, $(4,9,14,19,24,29)$ los adolescentes evaluados han respondido de manera escéptica los estudiantes del $7^{\circ}$ grado alcanzaron un porcentaje del $71 \%$ dentro del parámetro normal los varones, el $22 \%$ de los varones alcanzan el nivel superior y un $7 \%$, en el rango inferior. En la misma dimensión, las mujeres han indicado 58\% dentro del nivel normal, y $33 \%$ en el rango superior, el resto clasifica en $8 \%$ nivel inferior, superior y $7 \%$ en el nivel inferior. En el $8^{\circ}$ grado la distribución de los resultados se pudo discriminar en los estudiantes del sexo masculino el $77 \%$ clasifica en el rango normal, el $19 \%$ en el nivel superior, y el $4 \%$ en el rango inferior. En el mismo grado las estudiantes del sexo femenino han respondido el $74 \%$ dentro del rango normal $21 \%$ en el nivel superior y $5 \%$ dentro del rango inferior. Evaluando el $9^{\circ}$ los estudiantes del sexo masculino clasifican, dentro del rango normal $55 \%$ y el $38 \%$ en el nivel superior, y $6 \%$ en el rango inferior.

La DIMENSIÓN FÍSICA, en los estudiantes del sexo masculino del $7^{\circ}$ grado los resultados se encuentran dentro del nivel superior $50 \%$ los varones y las mujeres $37 \%$ y 
término normal; los varones $36 \%$ y las mujeres $40 \%$, en esta dimensión los varones en un $36 \%$ en el rango normal y $14 \%$ dentro del rango inferior. En el $8^{\circ}$ grado prepondera los resultados en la clasificación Normal. De igual forma, los datos del $8^{\circ}$ y $9^{\circ}$ grado presentan frecuencias similares. Los estudiantes del $8^{\circ}$ del sexo masculino se ubican en la posición del nivel superior en un 50\% y las mujeres en un $29 \%$ en el rango normal los varones señalan en un $35 \%$ y las mujeres en un $50 \%$ y en el nivel inferior los varones en un $15 \%$ y las mujeres en un $21 \%$. En el $9^{\circ}$ grado se evidencia en el rango superior $49 \%$ los estudiantes varones y en un $29 \%$ en las estudiantes mujeres, dentro del rango normal los varones en un $34 \%$ y las mujeres en un $49 \%$ y en la categoría inferior los estudiantes varones en un $17 \%$ y las mujeres en un $23 \%$.

\section{CONCLUSIÓN O CONSIDERACIONES FINALES}

De esta manera se enfatiza globalmente, que en los resultados del AF -5 , de la muestra estudiada, del $7^{\circ}, 8^{\circ}$ y $9^{\circ}$ grado de la EEB del Centro Regional de Educación de PilarParaguay, año 2019, en la destacan dos de las dimensiones; AF -1 (académico) y AF - 5 (Físico), en el aspecto académico, las mujeres presentan un nivel superior en relación a los varones. En tanto, en la dimensión AF - 5 se observa la prevalencia en el sexo masculino, dentro de los indicadores de aspecto físico, los varones manifiestan un nivel superior en el autoconcepto físico. Analizando los resultados en las otras dimensiones, se infiere niveles dentro de los parámetros normales en ambos sexos. Se debe de considerar que los adolescentes han participado y respondido de acuerdo a sus intereses e interrelaciones sociales, familiares. En el aspecto emocional se observó a los estudiantes escépticos en su mayoría no pretendían exteriorizar en cuanto a sus estados emocionales y sentimientos.

\section{LISTA DE REFERENCIAS}

Antolín Suárez, L., Oliva Delgado, A., Pertegal Vega, M. Á., \& López Jiménez, A. M. (2011). Desarrollo y validación de una escala de valores para el desarrollo positivo adolescente. Psicothema, vol. 23, núm. 1, 153-159.

Barrera Hurtado, M. D. (2012). Influencia de la Autoestima, el autoconcepto y la autoimagen social, en las Dimensiones de la Socialización de Estudiantes Universitarios. Caracas.

Bustos, V., Oliver, A., \& Galiana, L. (2015). Validación del Autoconcepto Forma 5 en Universitarios Peruano: Una herramienta para la Psicología Positiva2015. Psicología reflexión e crítica, 690 - 697. 
Cazalla Luna, N., \& Molero, D. (2013). Revisión Teórica sobre el Autoconcepto y su importancia en la adolescencia. Revista Electrónica de Investigación y Docencia (REID) 10, Julio, 43 - 64.

García, F., \& Musitu, G. (1999). AF-5 Autoconcepto Forma 5. . Madrid: TEA Ediciones S.A.

García, F., \& Musitu, G. (2014). Manual $4^{\circ}$ edición, revisada y ampliada. Madrid: TEA Ediciones.

Hernández Sampieri, R. (2014). Definición del alcance de la investigación que se realizará: exploratorio,descriptivo, correlacional o explicativo. E -uaem Espacio de Formación Multimodal, págs. 88-101.

Marulanda, A. (1999). Creciendo con nuestros hijos. Colombia: Cargraphics S.A.

Musitu, G., García, J., \& Gutiérrez, M. (1994). AF4 Autoconcepto Forma A. TEA(2).

Oliva, A., Pertegal, M. Á., Antolín, L., Reina, M. C., Ríos, M., Hernando, Á., . . . Estévez, R. M. (2010). El Desarrollo Positivo adolescente y los activos que lo promueven. Un estudio en Centros Docentes Andaluces. AndaLucia : Consejería de la Salud.

Reigal Garrido, R., Videra García, A., Parra Flores, J., \& Juárez Ruiz de Mier, R. (2012). Actividad físico deportiva, autoconcepto físico y bienestar psicológico en la adolescencia. RETOS. Nuevas Tendencias en Educación núm. 22, juliodiciembre, , 19-23.

Rodriguez, A., Goñi, A., \& Ruíz de Azúa, S. (2006). Autoconcepto físico y estilos de vida en la adolescencia. Psychosocial Intervention Vol $15 N^{\circ} 1$.

Shavelson , J., Hubner, J. J., \& Stanton, G. C. (1976). Self-concept: validation of construct interpretations. Revierw of Educational Research 46, 407 -442.

Tamayo y Tamayo, M. (2003). El proceso de investigación científica (cuarta ed.). México: LIMUSA, S.A. 